\title{
The TC10 -Exo70 Complex Is Essential for Membrane Expansion and Axonal Specification in Developing Neurons
}

\author{
Sebastián Dupraz, ${ }^{1}$ Diego Grassi, ${ }^{1}$ María Eugenia Bernis, ${ }^{1}$ Lucas Sosa, ${ }^{1,3}$ Mariano Bisbal, ${ }^{2}$ Laura Gastaldi, ${ }^{2}$ \\ Ignacio Jausoro, ${ }^{2}$ Alfredo Cáceres, ${ }^{2}$ Karl H. Pfenninger, ${ }^{3}$ and Santiago Quiroga ${ }^{1}$ \\ ${ }^{1}$ Departamento de Química Biológica y Centro de Investigaciones en Química Biológica de Córdoba, Facultad de Ciencias Químicas, Universidad \\ Nacional de Córdoba-Consejo Nacional de Investigaciones Científicas y Técnicas (CONICET), 5000 Córdoba, Argentina, ${ }^{2}$ Instituto de Investigación \\ Médica Mercedes y Martín Ferreyra-CONICET, 5016 Córdoba, Argentina, and ${ }^{3}$ Colorado Intellectual and Developmental Disabilities Research \\ Center and Department of Pediatrics, University of Colorado Denver, Aurora, Colorado 80045
}

Axonal elongation is one of the hallmarks of neuronal polarization. This phenomenon requires axonal membrane growth by exocytosis of plasmalemmal precursor vesicles (PPVs) at the nerve growth cone, a process regulated by IGF-1 activation of the PI3K (phosphatidylinositol-3 kinase) pathway. Few details are known, however, about the targeting mechanisms for PPVs. Here, we show, in cultured hippocampal pyramidal neurons and growth cones isolated from fetal rat brain, that IGF-1 activates the GTP-binding protein TC10, which triggers translocation to the plasma membrane of the exocyst component exo70 in the distal axon and growth cone. We also show that TC10 and exo70 function are necessary for addition of new membrane and, thus, axon elongation stimulated by IGF-1. Moreover, expression silencing of either TC10 or exo70 inhibit the establishment of neuronal polarity by hindering the insertion of IGF-1 receptor in one of the undifferentiated neurites. We conclude that, in hippocampal pyramidal neurons in culture, (1) membrane expansion at the axonal growth cone is regulated by IGF-1 via a cascade involving TC10 and the exocyst complex, (2) TC10 and exo70 are essential for the polarized externalization of IGF-1 receptor, and (3) this process is necessary for axon specification.

\section{Introduction}

The development of a typical polarized neuron, composed of one long axon and several branching dendrites, requires the action of two interrelated processes, neurite outgrowth and specification of the axon. The initial signals and pathways that determine polarity are beginning to be understood. A particularly early event, in neurons that do not yet exhibit a discernible axon (stage 2 of differentiation) (Sosa et al., 2006), is the segregation of activatable IGF-1 receptors in one neurite. Subsequently, phosphatidylinositol-3 kinase (PI3K) and its product, $\mathrm{PIP}_{3}$ (phosphatidylinositol 3,4,5-trisphosphate), accumulate in the distal region and growth cone of that neurite, together with the IGF-1 receptor. These events are critical for the outgrowth of the future axon (Shi et al., 2003; Ménager et al., 2004; Nishimura et al., 2005). Indeed, designation of the axon requires activation of PI3K by the IGF-1 receptor (Shi et al., 2003; Sosa et al., 2006). In addition to axonal specification, the establishment of polarity necessitates axonal elongation and, therefore, the addition of new membrane to the plasmalemma of the axon. Axolemmal expansion occurs by exocytosis of plasmale-

\footnotetext{
Received Aug. 10, 2009; accepted Sept. 3, 2009.

This work was supported by grants from the Agencia Nacional de Promoción Científica y Tecnológica, Argentina (S.Q., A.C.); Consejo Nacional de Investigaciones Científicas y Técnicas, Argentina (A.C., S.Q.); the National Institutes of Health (K.H.P.); and Secretaría de Ciencia y Técnica de la Universidad Nacional de Córdoba (S.Q.)

Correspondence should be addressed to Santiago Quiroga, Departamento de Química Biológica-Centro de Investigaciones en Química Biológica de Córdoba, Facultad de Ciencias Químicas, Universidad Nacional de CórdobaConsejo Nacional de Investigaciones Científicas y Técnicas, Haya de la Torre esquina Medina Allende, Ciudad Universitaria, 5000 Córdoba, Argentina. E-mail: squiroga@mail.fcq.unc.edu.ar. DOI:10.1523/JNEUROSCI.3907-09.2009

Copyright $\odot 2009$ Society for Neuroscience $\quad$ 0270-6474/09/2913292-10\$15.00/0
}

mmal precursor vesicles (PPVs) primarily at the neuronal growth cone (Pfenninger and Maylié-Pfenninger, 1981; Pfenninger and Friedman, 1993; Futerman and Banker, 1996), a process regulated by IGF-1 activation of the PI3K pathway (Pfenninger et al., 2003; Laurino et al., 2005; Pfenninger, 2009).

The exocyst complex is thought to be critical for several forms of polarized exocytosis (TerBush et al., 1996). In yeast, it marks regions of membrane addition during budding and cytokinesis (TerBush and Novick, 1995; Finger et al., 1998; Guo et al., 1999). In multicellular organisms, the exocyst complex has been implicated in a variety of processes involving exocytosis, such as the establishment of polarity in epithelial cells (Grindstaff et al., 1998; Yeaman et al., 2001), the insulin-regulated insertion of Glut4, the glucose transporter, into the plasmalemma of adipocytes (Inoue et al., 2003), and postsynaptic NMDA and AMPA receptor trafficking (Sans et al., 2003). In hippocampal neurons, a widely used system to study neuronal growth and polarization, exocyst subunits (the Sec6/8 complex) are enriched at growth cones (Hazuka et al., 1999). In PC12 cells, neurite outgrowth is repressed by a deletion mutant of the exocyst component Sec10 (Vega and Hsu, 2001), and in adipocytes yet another exocyst protein, exo70, translocates to the plasma membrane in response to insulin. This occurs via activation of the small GTPase TC10, which assembles a multiprotein complex including Sec6 and Sec8 at the plasmalemma (Inoue et al., 2003). An NGF-induced exo70-TC10 complex has been shown to modulate neurite outgrowth in PC12 cells (Pommereit and Wouters, 2007).

In this study, we examined the roles of exo70 and TC10 in axonal membrane biogenesis and growth of differentiating hip- 

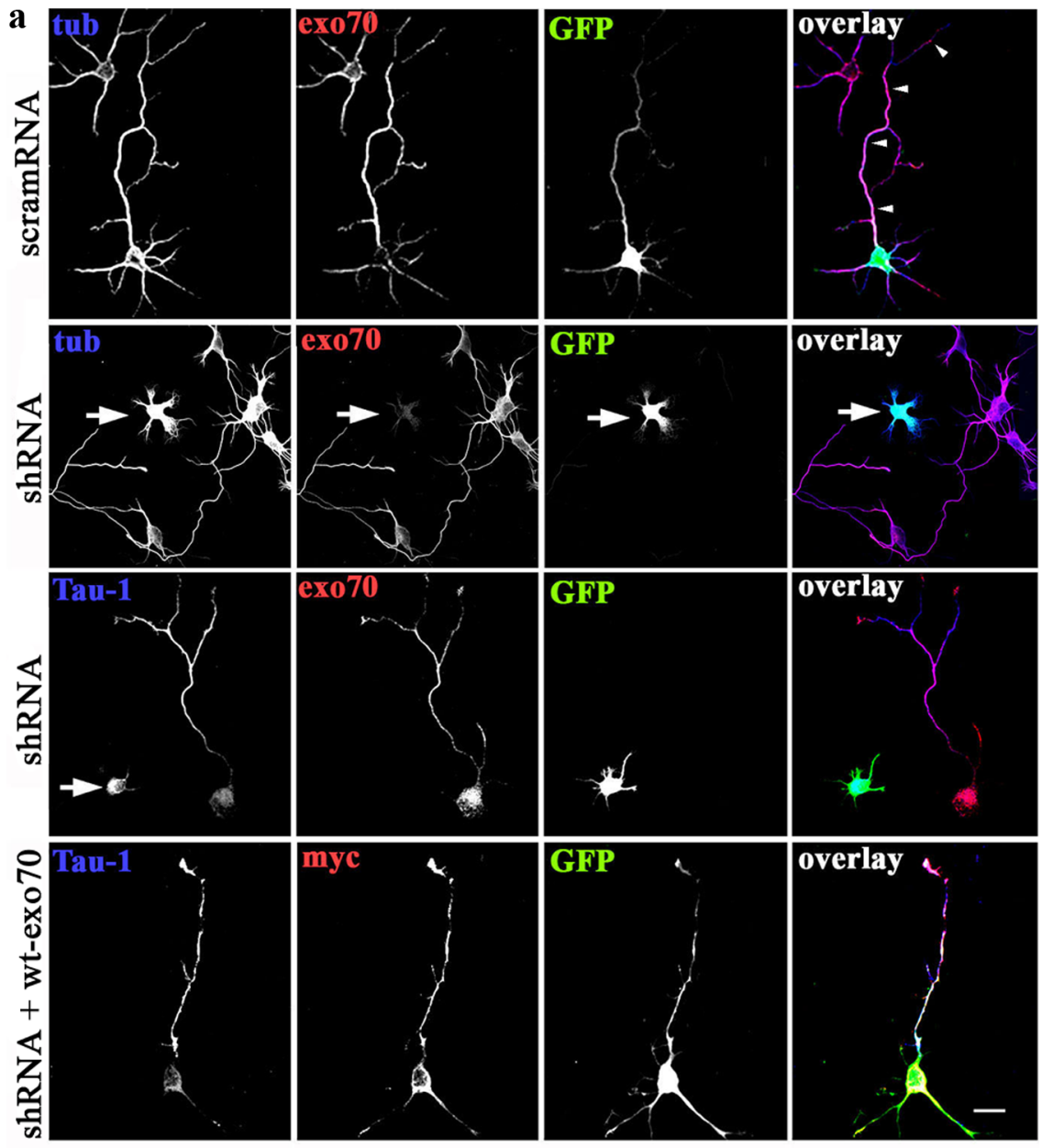

b

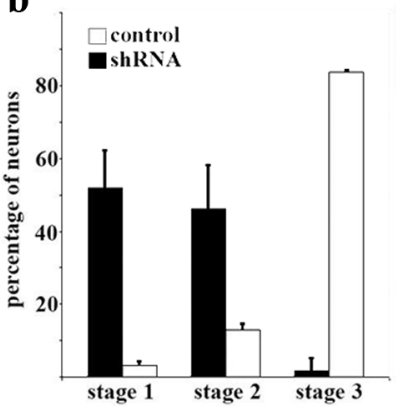

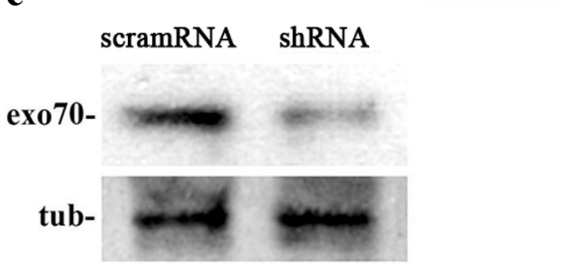

\section{Materials and Methods}

Short hairpin RNA plasmids. Plasmids containing short hairpin RNA (shRNA) were constructed in $\mathrm{pBS} / \mathrm{U} 6$ vectors according to the procedures described previously (Xia et al., 2003). cDNAs encoding shRNAs were inserted in a pCAGIG vector in which the green fluorescent protein (GFP)-cDNA is under the control of chick actin-minimal cytomegalovirus (CMV) (CAG) promoter (the pBS/U6 and pCAGIG vectors were a generous gift from Dr. C.-H. Sung, Weill Medical College of Cornell University, New York, NY). The DNA sequences used as targets were as follows: Exo70, ggagaatgtcgaaaagacc (a generous gift from Dr. R. Prekeris, School of Medicine, University of Colorado Denver, Aurora, CO); Tc10, gggtaccagaactaaaggaat [similar to the sequence used by Kawase et al. (2006)], and, for controls, gggtaaccaatagaggaca, a scrambled sequence that was created using the Wizard design web page (InVivogen). The resulting plasmids were referred to as Exo70-shRNA, TC10-shRNA, and scramRNA (scrambled shRNA), respectively.

DNA constructs. The coding sequence of rat TC10 was PCR amplified from a rat brain cDNA library using forward primer $5^{\prime}$-cggcgaattcatggctcacgggccc- $3^{\prime}$ and reverse primer $5^{\prime}$ ggatgtcgacgacaggctccctcccc- $3^{\prime}$. The PCR product was cloned into the EcoRI/SalI sites of a pCMV vector (Clontech) that contained the sequence encoding the Myc or hemagglutinin (HA) tag at the $5^{\prime}$ end of the cloning site. To construct the dominant-negative $(\mathrm{T} 23 \mathrm{~N})$ mutant form of TC10, the wild-type construct was mutated using the QuikChange site-directed mutagenesis kit (Stratagene). Complementary primers on the site of mutagenesis for $\mathrm{T} 23 \mathrm{~N}$ were $5^{\prime}$-ggggcggtgggtaagaactgcctgctcatgagc- $3^{\prime}$ and its complementary strand. The coding sequence of rat Exo70 was PCR amplified from a rat brain cDNA library using forward primer $5^{\prime}$ gcgctgtccgaattcatgattccccegcagg- $3^{\prime}$ and reverse primer $5^{\prime}$-gggcggaagatctggccgacttaagcagagg- $3^{\prime}$. The PCR product was cloned into the EcoRI/BgIII sites of the PCMV vector (Clontech) containing a Myc or HA tag at the $5^{\prime}$ end of the cloning site. The GFP-tagged L1 construct was a generous gift from Dr. T. Galli (Inserm U950, University Denis Diderot, Paris, France) (Dequidt et al., 2007).

Primary antibodies. The following primary antibodies were used: affinity-purified rabbit polyclonal antibody to exo70 (generous gift from Dr. R. Prekeris), diluted 1:1000 for immunofluorescence (IF) and 1:2000 for Western blot (WB); goat polyclonal antibodies to TC10 (E-13 and A-14; Santa Cruz Biotechnology), diluted 1:50 (IF) or 1:250 (WB); goat polyclonal anti-GAP-43 (C-19; Santa Cruz Biotechnology), diluted 1:250; rabbit polyclonal antibody to $\beta$ III-tubulin (Sigma-Aldrich), diluted 1:2000; rat monoclonal antibody to tyrosinated $\alpha$-tubulin (clone Tub-IA2; SigmaAldrich), diluted 1:2000; mouse monoclonal and rabbit polyclonal antibodies to c-Myc (Sigma-Aldrich), diluted 1:400; rat monoclonal antibody to HA (clone 3F10 Roche), 1:700; mouse monoclonal antibody to the axonal marker tau-1 (Calbiochem), diluted 1:200; mouse monoclonal anti-GFP (Roche), diluted 1:600. pocampal pyramidal neurons in culture. In these cells, loss of function of either TC10 or exo70 repressed not only axonal membrane expansion and outgrowth but also the establishment of neuronal polarity by inhibiting the polarized insertion of IGF-1 receptor in one of the minor neurites. 
Culture and transfection. Dissociated hippocampal pyramidal neurons were prepared from fetal rat brain and cultured as described previously (Rosso et al., 2004). In brief, cells were plated onto polylysine-coated glass coverslips and maintained in DMEM plus 10\% horse serum for $1 \mathrm{~h}$. The coverslips with the attached cells were transferred subsequently to $60 \mathrm{~mm}$ Petri dishes containing serum-free medium plus the N2 mixture. Cultures were maintained in a humidified $37^{\circ} \mathrm{C}$ incubator with $5 \% \mathrm{CO}_{2}$. Shortly after plating, hippocampal neurons first extend lamellipodia (stage 1) and afterward several minor neurites that are initially indistinguishable (stage 2). Then, at stage 3 , one of these initially equivalent neurites grows more rapidly than the others and becomes the axon, whereas the other neurites subsequently develop into dendrites (stage 4). Neurons are considered to be at stage 3 when the length of the axon exceeds that of the average minor neurite by at least $20 \mu \mathrm{m}$ (Craig and Banker, 1994). Transient transfection of cultured neurons was performed as described previously (Rosso et al., 2004), and the constructs used at a concentration of $2 \mu \mathrm{g} / \mu \mathrm{l}$.

Immunofluorescence microscopy. Cells were fixed for $1 \mathrm{~h}$ at room temperature with $4 \%$ (w/v) paraformaldehyde in PBS containing 4\% $(w / v)$ sucrose. Cultures were washed with PBS, permeabilized with $0.1 \%(\mathrm{v} / \mathrm{v})$ Triton X-100 in PBS for $6 \mathrm{~min}$, and again washed in PBS. After labeling with a first primary antibody $(1-3 \mathrm{~h}$ at room temperature) and washing with PBS, cultures were incubated with fluorescent secondary antibody (conjugated to Alexa Fluor 488, 546 , or $633 ; 1 \mathrm{~h}$ at $37^{\circ}$ ) and washed with PBS. The same procedure was repeated for the second and third primary and secondary antibodies. For the experiments using the L1-GFP construct (see Fig. 6), cells were fixed as described previously and labeled with the antiGFP and a fluorescent secondary antibody (conjugated to Alexa Fluor 546). Afterward, cells were permeabilized as described previously and labeled with the anti-Myc antibody and the corresponding secondary antibody.

The cells were observed with a Zeiss Pascal 5 confocal microscope. Images were captured and digitized using LSM Image software. For some experiments (see Figs. 4, 5), cells were observed with a Nikon total internal reflection fluorescence (TIRF) microscope. Images were captured using a CCD camera (Hamamatsu) and digitized directly into a MetaMorph/Metafluor Image Processor (Universal Imaging). All images were printed using Adobe PhotoShop.

Gel electrophoresis and Western blot. Proteins were separated by SDSPAGE. The concentration of acrylamide of the resolving gel varied from 7.5 to $11 \%$. The resolved proteins were transferred to polyvinylidene difluoride membranes in Tris-glycine buffer containing 20\% methanol. The membranes were first dried, washed with Tris-buffered saline (TBS) (10 mm Tris, $\mathrm{pH} 7.5,150 \mathrm{~mm} \mathrm{NaCl}$ ) and then blocked, or directly blocked for $1 \mathrm{~h}$ in TBS containing 5\% BSA. The blots were incubated with the primary antibodies in PBS containing 0.05\% Tween 20, for $2 \mathrm{~h}$ at room temperature. After washing with TBS containing 0.05\% Tween 20, the membranes were incubated with horseradish peroxidase-conjugated secondary antibodies (Sigma-Aldrich) for $1 \mathrm{~h}$ at room temperature. After washing, the blots were developed using a chemiluminescence detection kit (ECL; GE Healthcare Life Sciences).

Isolation of growth cones. Axonal growth cones were isolated from developing brain as described previously (Pfenninger et al., 1983; Lohse et al., 1996). In brief, brains of $18 \mathrm{~d}$ gestation fetal rats were homogenized (H). A low-speed supernatant (LSS) was prepared, loaded onto a discontinuous sucrose density gradient with steps of 0.83 and $2.66 \mathrm{~m}$ sucrose, and spun to equilibrium at $242,000 \times g_{\max }$. The fraction at the load/0.83 $\mathrm{M}$ interface (designated " $\mathrm{A}$ ") contained the isolated growth cones or growth cone particles (GCPs).

TC10 activity assays. GCPs (prepared as described previously) were incubated in control medium, medium containing $10 \mathrm{~nm}$ IGF-1, $10 \mathrm{~nm}$ IGF-1 plus $20 \mu \mathrm{M}$ 2-(4-morpholinyl)-8-phenyl-4 H-1-benzopyran-4one (LY294002), or $50 \mathrm{~nm}$ insulin for $2 \mathrm{~min}$. Lysed GCPs were incubated with binding buffer ( $25 \mathrm{~mm}$ Tris- $\mathrm{HCl}, \mathrm{pH} 7.5,1 \mathrm{~mm}$ dithiothreitol, $30 \mathrm{~mm}$ $\mathrm{MgCl}_{2}, 40 \mathrm{mM} \mathrm{NaCl}$, and $0.5 \%$ Nonidet P-40) in the presence of $7 \mathrm{mg}$ of agarose-conjugated glutathione $S$-transferase (GST)-Pak1 p21-binding domain (Millipore) for $1 \mathrm{~h}$ at $4^{\circ} \mathrm{C}$. The beads were washed three times with $1 \%$ Nonidet P-40 washing buffer, and TC10 levels were determined by immunoblot as described previously.

Exo70 and TC10 rescue experiments. To perform these experiments, we titrated levels of exo70 or TC10 plasmid to determine the lowest concentration necessary to overcome the shRNA effects. Under these conditions, cells transfected with exo70 or TC10 shRNA, together with exo70 or TC10 plasmid, respectively, exhibited protein levels that were similar to those of control nontransfected neurons (supplemental Figs. 2, 3, available at www.jneurosci.org as supplemental material). 

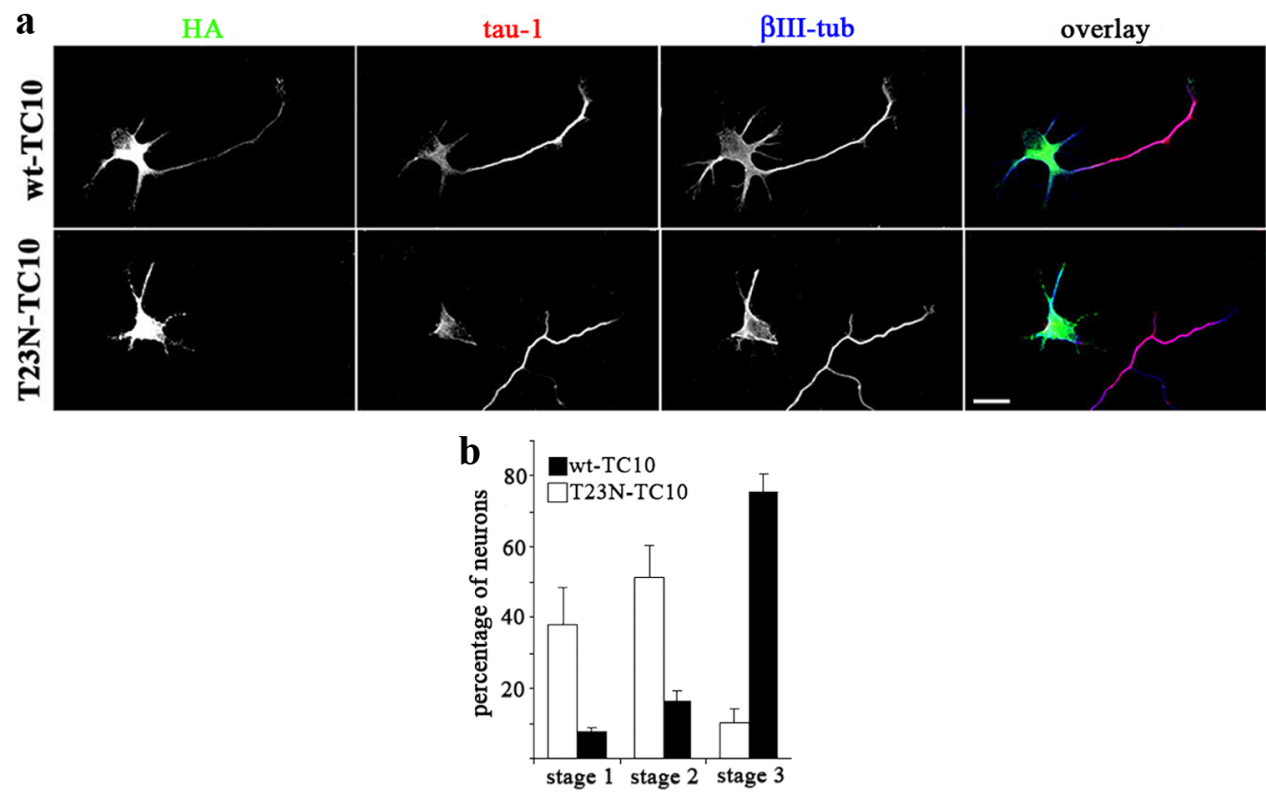

Figure 3. Dominant-negative (T23N) TC10 inhibits neuronal polarization. $\boldsymbol{a}$, Hippocampal pyramidal neurons were transfected with HA-tagged wild-type TC10 (wt-TC10) or T23N-TC10. Triple immunofluorescence shows HA antigen (green), tau-1 (red), and the neuronal marker $\beta$ III-tubulin (blue). The neuron transfected with T23N-TC10 did not develop an axon and developed only short, minor neurites. Scale bar, $20 \mu \mathrm{m}$. $\boldsymbol{b}$, Percentages (+SEM) of control neurons or neurons containing T23N-TC10 at specific stages of differentiation after $36 \mathrm{~h}$ in culture. $n=3$ independent experiments. At least 50 neurons were scored for each condition.

Time-lapse total internal fluorescence microscopy. For time-lapse TIRF microscopy, cells were cultured in special Petri dishes (Paglini et al., 1998). Twelve hours after transfection with either wild-type TC10 or the dominant-negative form T23N-TC10 plus L1-GFP, the dishes containing the attached cells (deprived of growth factors for the last $4 \mathrm{~h}$ ) were placed in a Harvard microincubator located on top of the stage of a fully motorized Nikon TE-2000 E inverted microscope equipped for differential interference contrast, epifluorescence, and TIRF. We used TIRF to image single vesicular insertion events under low-intensity conditions that minimize phototoxicity (Yudowski et al., 2006). Cells were visualized with a $60 \times, 1.45$ numerical aperture objective, equipped for through-the-objective TIRF illumination using a $488 \mathrm{~nm}$ argon laser. Neurons were imaged in Neurobasal medium supplemented with $20 \mathrm{~nm}$ IGF- 1 and $30 \mathrm{~mm}$ HEPES buffer, $\mathrm{pH} 7.2$, and maintained at $37^{\circ} \mathrm{C}$. Timelapse sequences were acquired at a continuous rate of 1 frame/s during 4 min using an ORCA II-ER (Hamamatsu) camera and MetaMorph software (Molecular Devices). Pyramidal neurons were selected by morphological criteria (in wide-field images) before imaging in TIRF mode. Live-cell images shown represent raw data with simple background subtraction of the averaged blank field intensity. After time-lapse imaging, the cultures were fixed and stained with anti-myc antibody to identify cells transfected with either wt-TC10 or T23N-TC10. Only the growth cones from transfected cells were scored for quantification.

Animals. All animal procedures were done using approved protocols by the Board of Animal Welfare, School of Chemical Sciences, National University of Córdoba.

\section{Results}

Distribution and role of TC10 and exo70 in neurite growth We first investigated the expression and subcellular distribution of exo70 and TC10 in different subcellular fractions of fetal brain, especially in the primarily axonal growth cone fraction (Pfenninger et al., 1983; Lohse et al., 1996). Results showed that exo70 was highly enriched in isolated growth cones (GCPs) (supplemental Fig. $1 A$, available at www.jneurosci.org as supplemental material) compared with brain homogenate $(\mathrm{H})$, low-speed supernatant (LSS), and fraction A (A), which consists of GCPs suspended in cytosolic proteins from the homogenate (Pfenninger et al., 1983). TC10 was particularly enriched in fraction A but also concentrated in GCPs with respect to brain homogenate $(\mathrm{H})$ (supplemental Fig. $1 A$, available at www.jneurosci.org as supplemental material). The distribution of GAP43, a protein highly enriched in GCPs (Lohse et al., 1996), is shown for comparison. We also analyzed the expression and distribution of exo70 and TC10 in primary cultures of hippocampal neurons after $24 \mathrm{~h}$ of differentiation in vitro (Mascotti et al., 1997). Consistent with the fractionation data, exo70 immunostaining was enriched in the distal one-third of the axon and the growth cone of neurons at stage 3 of differentiation (supplemental Fig. $1 B$, available at www. jneurosci.org as supplemental material). Immunostaining with an anti-TC10 antibody showed that, unlike exo70, this small GTP-binding protein was present in the perikaryon, the minor neurites, the axonal shaft, and the growth cone of the hippocampal neurons (supplemental Fig. $1 B$, available at www.jneurosci. org as supplemental material). In the distal axon and growth cone (including the leading edge), a prominent site of new membrane addition in developing neurons (Pfenninger and MayliéPfenninger, 1981; Craig et al., 1995; Pfenninger et al., 2003; Laurino et al., 2005), exo70 and TC10 were colocalized (supplemental Fig. $1 B, C$, available at www.jneurosci.org as supplemental material).

The IGF-1 receptor controls the establishment of neuronal polarity by activating the $\mathrm{PI} 3 \mathrm{~K}-\mathrm{Akt}-\mathrm{Cdc} 42$ pathway and also regulates addition of new membrane at the axonal growth cone of developing neurons. To study the possible involvement of exocyst complex components, we silenced expression of such proteins using targeted shRNAs inserted into dicistronic plasmids also encoding enhanced GFP. Transfection of hippocampal neurons in culture with exo70-targeted shRNA significantly and specifically decreased exo70 protein in the cultures (Fig. 1c). The transfected neurons expressed virtually no detectable exo70 and failed to form axons; only short, minor neurites were present (Fig. $1 a$, second row from top, arrow). Note also the lack of enrichment of tau- 1 in any neurite of the transfected neurons (Fig. $1 a$, third row from top, arrow). In contrast, neurons transfected with a scram- 
bled RNA sequence inserted in the same plasmid exhibited normal levels of exo70 and generated a long axon-like process (Fig. $1 a$, top, arrowheads). Cotransfection of neurons with exo70-targeted shRNA and a myc-tagged wild-type form of exo70 rescued the phenotype and induced the outgrowth of an axon-like process enriched in tau-1 protein (Fig. $1 a$, bottom). The levels of exo70 expression (as revealed by immunocytochemistry) in control nontransfected cells, shRNA transfected cells, and shRNA plus wt-exo70 transfected cells are shown in supplemental Figure 2 (available at www.jneurosci.org as supplemental material). Expression of exo70 in the cotransfected cells is similar to that in control nontransfected cells (see also Materials and Methods). To analyze this observation quantitatively, we scored the differentiation stages of neurons transfected with exo70-targeted shRNA compared with neurons in the same cultures not containing shRNA, after $48 \mathrm{~h}$ in vitro. We found that $>95 \%$ of the transfected neurons remained at stages 1 or 2 of differentiation, and $<5 \%$ had formed a discernible axon. In contrast, $>80 \%$ of the control neurons showed an identifiable, tau-1-containing axon (Fig. 1b). We performed similar experiments with shRNA targeting TC10, the exo70 activator. Western blots showed that transfection with the dicistronic plasmid containing TC10-targeted shRNA plus GFP significantly reduced TC10 expression in hippocampal neurons in culture (Fig. 2c). TC10 was not detectable by immunofluorescence in transfected neurons. Such neurons failed to form an axon and generated only short, minor neurites (Fig. 2a, top). These neurons also failed to polarize tau-1 protein to any process (Fig. $2 a$, middle), whereas growing axons from nontransfected neurons in the same culture were clearly tau-1-positive (Fig. $2 a$, middle, arrowheads). Cotransfection with TC10 shRNA plus a myctagged wild-type form of TC10 rescued the phenotype and generated neurons with axon-like processes enriched in tau-1 protein (Fig. $2 a$, bottom). The levels of TC10 expression (revealed immunocytochemically) in control nontransfected cells, shRNA-transfected cells, and shRNA plus wt-TC10-transfected cells are shown in supplemental Figure 3 (available at www. jneurosci.org as supplemental material). The expression of TC10 in the cotransfected cells is similar to that in control nontransfected neurons (see also Materials and Methods). As in the case of exo70, most neurons transfected with TC10-targeted shRNA remained at stages 1 and 2 of differentiation (Fig. 2b). Additional evidence for the participation of TC10 in neurite outgrowth and the establishment of neuronal polarity was obtained by transfecting hippocampal neurons with a dominant-negative form of TC10 (T23N). After $36 \mathrm{~h}$ in culture, neurons transfected with wild-type TC10 (myctagged) differentiated apparently normally and grew a long, axonlike process (Fig. 3a, top). In contrast, neurons transfected with
T23N-TC10 remained at stage 2 of differentiation, with only short, minor neurites without detectable tau-1 levels (Fig. $3 a$, bottom). Quantitative data are shown in Figure $3 b$. The levels of expression of endogenous TC10 and T23N-TC10 are shown immunocytochemically in supplemental Figure 4 (available at www.jneurosci.org as supplemental material) and indicate that the mutant construct is not expressed at a very high, potentially toxic level. Together, these results indicate that the TC10-exo70 complex is essential for axonal growth and suggest that it is involved in the establishment of polarity in hippocampal neurons.

\section{TC10 activation and exo70 recruitment by IGF-1}

Insulin-regulated translocation of Glut 4 from intracellular storage sites to the plasma membrane requires activation of TC10 in adipocytes and muscle cells (Chiang et al., 2001). Although insulin receptors are essentially absent from growth cones of differentiating neurons, IGF-1 receptors are enriched significantly (Quiroga et al., 1995; Mascotti et al., 1997; Sosa et al., 2006). Therefore, we investigated whether TC10 was activated by IGF-1 in growth cones. We stimulated isolated growth cones (GCPs) 


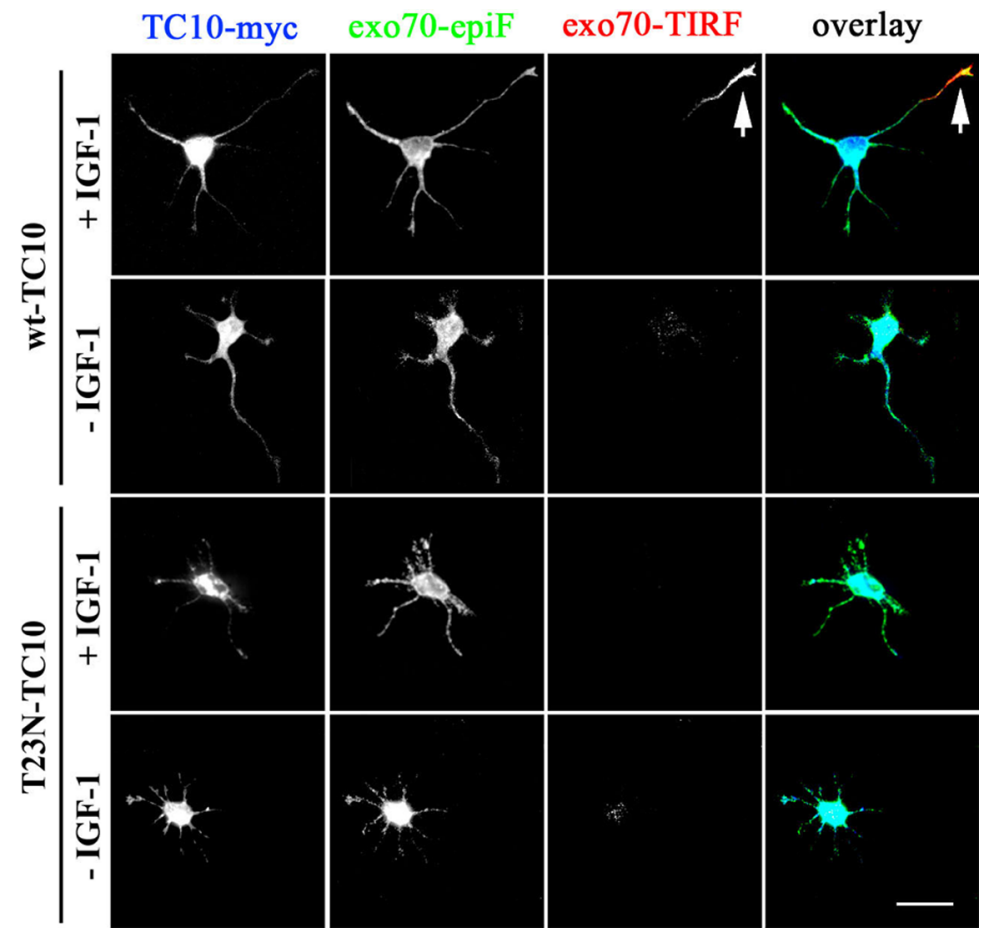

Figure 5. IGF-1-triggered association of exo70 with the plasmalemma requires functional TC10. Double-immunofluorescence micrographs of hippocampal neurons cultured for $24 \mathrm{~h}$ show the distributions of myc-tagged wild-type or dominant-negative (T23N) TC10 (blue), and of ex070, observed by epifluorescence (green) or TIRF microscopy (red). Neurons were stimulated for 2 min in control or $10 \mathrm{~nm} \mathrm{IGF-1} \mathrm{medium.} \mathrm{Plasmalemmal} \mathrm{enrichment} \mathrm{of} \mathrm{exo} 70$ at the growth cone and in the distal axon is evident after IGF-1 stimulation (arrow), but not in the presence of T23N-TC10. Scale bar, $20 \mu \mathrm{m}$.

with $10 \mathrm{~nm}$ IGF-1 for $2 \mathrm{~min}$, solubilized them, and affinitypurified the GTP-bound form of TC10 on GST-agarose containing the Cdc42 binding domain of Pak1 (Chiang et al., 2001). TC10 levels were determined by Western blot. These blots showed that the percentage of GTP-bound (activated) TC10 relative to total TC10 was increased significantly $(p \leq 0.001)$ by IGF-1, compared with control (unstimulated) GCPs, insulinstimulated GCPs, or GCPs stimulated with IGF-1 in the presence of $20 \mu \mathrm{M}$ LY294002 (Fig. $4 a$; numerical values shown below blots). Inhibition of TC10 activation by LY294002 suggested that PI3K was necessary for TC10 activation by IGF-1. In other cell systems, activation of TC10 results in translocation of exo70 to the plasma membrane (Inoue et al., 2003). This also applied to growth cones (Fig. 4b). Exo70 was below detection levels in the soluble fraction $(\mathrm{S})$ in control GCPs, and this did not change with IGF-1 stimulation. Instead, most exo70 was associated with membrane $(\mathrm{M})$ and cytoskeletal $(\mathrm{C})$ fractions prepared from GCPs. Ten nanomolar IGF-1 raised the association of exo70 with the membrane fraction significantly so that the membrane/cytoskeleton ratio of exo70 increased over fourfold relative to that in control, unstimulated, GCPs.

We also studied exo70 association with the plasma membrane in intact hippocampal pyramidal neurons in culture. After control incubation or challenge with $10 \mathrm{nM}$ IGF-1 for $2 \mathrm{~min}$, the cultures were fixed, permeabilized, immunostained with an antibody to exo70, and analyzed using epifluorescence or evanescent-wave fluorescence (TIRF) microscopy. TIRF microscopy allows selective imaging of fluorescent probes located in close proximity $(<100 \mathrm{~nm})$ to the coverslip on which the cells grow (i.e., associated with the adherent plasma membrane). Although epifluorescence revealed similar levels of exo70 in control and IGF-1-stimulated neurons and processes, the TIRF images differed (Fig. 4c). In control neurites, TIRF detected essentially no exo70, whereas neurons challenged with IGF-1 exhibited a strong exo70 TIRF signal in the distal one-third of the axon and growth cone, where most membrane addition occurs in developing neurons (Fig. $4 c$ ). For comparison, we analyzed the membrane association of the synaptic vesicle protein synaptophysin in neurons under similar experimental conditions. The results indicated that challenging with IGF-1 did not cause any noticeable increment of synaptophysin TIRF signal at the growth cone of the neurons (supplemental Fig. 5, available at www.jneurosci.org as supplemental material). To correlate exo70 recruitment to the plasma membrane with TC10 activity, we performed similar experiments in neurons transfected with wild-type or dominant-negative forms of TC10. In neurons transfected with wild-type TC10 and challenged with IGF-1, TIRF detected the expected increment of exo70 in the distal axon and growth cone (Fig. 5, top, arrow). Transfection with dominant-negative T23NTC10, however, abolished IGF-1-triggered membrane recruitment of exo70 as seen by TIRF (Fig. 5, bottom).

\section{TC10 and membrane expansion}

To establish a direct link between TC10 activity and membrane addition at the growth cone, we used as a membrane marker the cell-adhesion molecule L1 fused at its N terminus to GFP (L1GFP) via a linker cleavable with thrombin (Dequidt et al., 2007). For experimentation, such cultures were treated with thrombin to remove externally exposed GFP. Monitoring subsequent recovery of GFP at the cell surface allowed us to assess insertion of new membrane containing L1-GFP. We cotransfected hippocampal neurons (after $24 \mathrm{~h}$ in vitro) with L1-GFP and either wild-type TC10 or T23N-TC10; 12 h later, the cells were treated for $100 \mathrm{~s}$ with thrombin and allowed to recover for $3 \mathrm{~h}$ in control conditions or after stimulation with $10 \mathrm{~nm} \mathrm{IGF-1.}$

Immunostaining of nonpermeabilized cells with an anti-GFP antibody revealed the L1-GFP inserted into the plasmalemma during the recovery period. Results of these experiments are shown in Figure $6 a$. They indicated that restoration of extracellular L1-GFP in the growth cone and distal axon was detectable only in those neurons that were transfected with wild-type TC10 and stimulated with IGF-1 (Fig. $6 a$, top). In contrast, the cells transfected with the dominant-negative T23N-TC10 exhibited no appreciable differences in extracellular GFP labeling between control neurons and IGF-1-stimulated neurons (Fig. $6 a$, bottom). For quantitative analysis, we measured the fluorescence intensity of surface GFP (as revealed by immunostaining of nonpermeabilized cells with anti-GFP antibody) and of total GFP in the distal one-third of the axon and growth cone of these neurons. The ratios of surface GFP/total GFP fluorescence intensity are shown in Figure $6 b$. They indicate that treatment with IGF-1 did not significantly increase surface GFP in the cells transfected with dominant-negative T23N-TC10. In the cells transfected with wild-type TC10, however, treatment with IGF-1 triggered an almost fourfold, significant increment in the ratio of surface/total GFP. Neurons mock-transfected with scrambled 


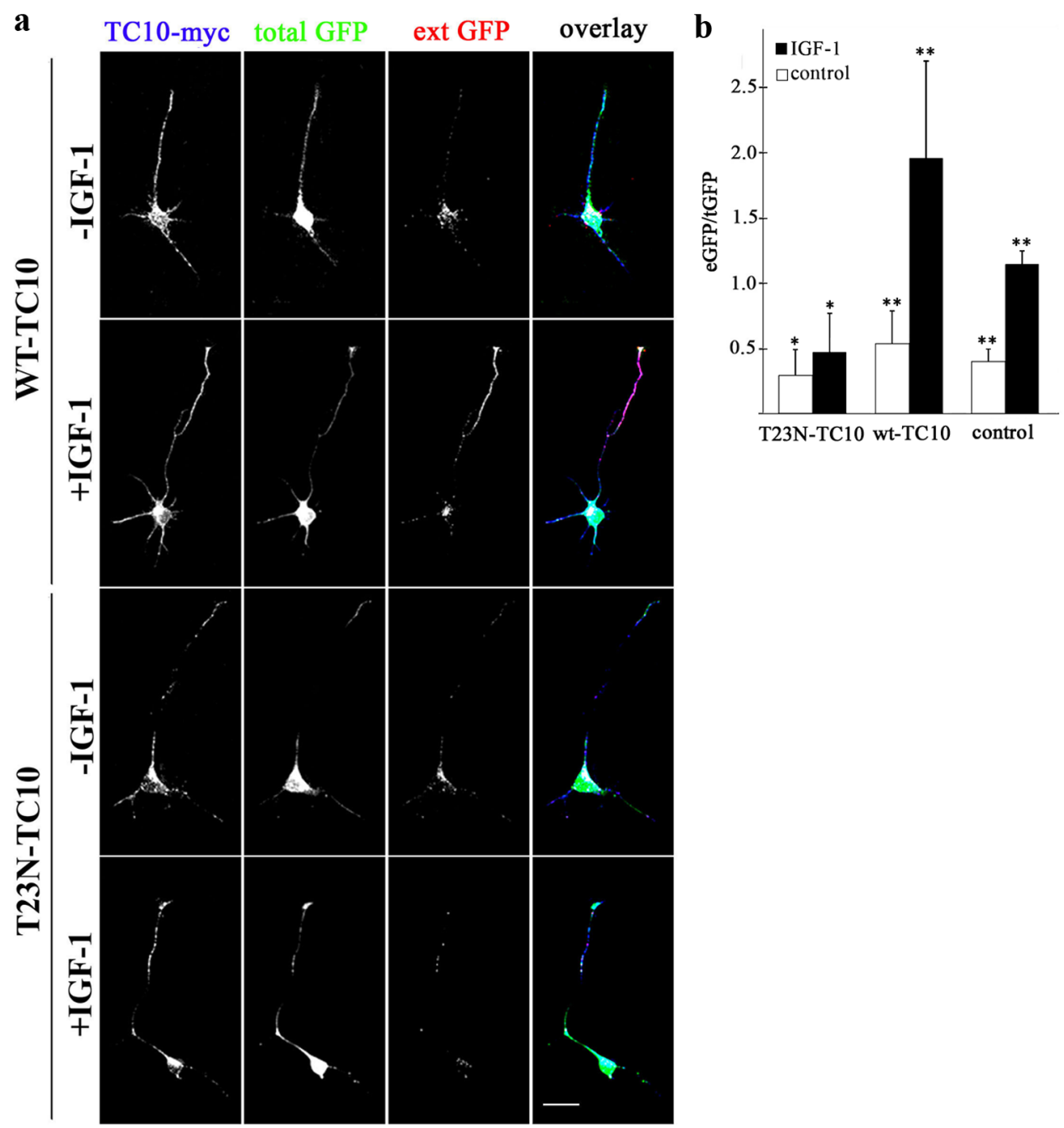

Figure 6. IGF-1-stimulated plasmalemmal insertion of L1-GFP requires functional TC10. $a$, Triple-fluorescence micrographs of hippocampal neurons cultured for $36 \mathrm{~h}$, treated with thrombin to remove exposed GFP, and then incubated for $3 \mathrm{~h}$ in control medium or medium containing $10 \mathrm{~nm}$ IGF-1. Blue, myc-tagged wild-type or dominant-negative (T23N) TC10; green, L1-GFP; and red, externally exposed GFP (antibody label without permeabilization). Note the substantial increment in external GFP immunoreactivity at the growth cone and distal axon of the neurons stimulated with IGF-1 in the presence of wild-type TC10 (second row). In contrast, neurons transfected with T23N-TC10, with or without IGF-1 stimulation, did not exhibit any detectable L1-GFP on the cell surface (third and fourth rows). $\boldsymbol{b}$, Ratios of external GFP (eGFP; red)/total GFP (tGFP; green) fluorescent intensity (arbitrary units) of distal axons and growth cones processed as shown in $\boldsymbol{a}$. IGF-1 significantly increased the eGFP/tGFP ratio in the neurons transfected with wild-type TC10 or control neurons transfected with L1-GFP $\left({ }^{* *} p<0.001\right)$, but not in those transfected with dominant-negative TC10 ( $\left.{ }^{*} \mathrm{NS}\right)$. Error bars indicate SEM. Scale bar, $20 \mu \mathrm{m}$.

RNA and treated with IGF-1 also exhibited a significant increment of this ratio. To establish a direct relationship between TC10 activity and PPV exocytosis at the growth cone of differentiating hippocampal neurons, we used time-lapse TIRF microscopy (Bisbal et al., 2008) to evaluate whether or not transfection with T23N-TC10 reduced the number of fusion events of L1-GFP-containing vesicles with the growth cone plasmalemma. Figure 7 shows two examples of such fusion events at the growth cone of a hippocampal pyramidal neuron transfected with wt-TC10 plus L1-GFP. The estimated frequency of fusion events at the growth cone of neurons transfected with wt-TC10 plus L1-GFP was $1.2+$ 0.4 min $^{-1}$ growth cone $e^{-1}(n=11)$ compared with $0.08+0.04$ $\min ^{-1}$ growth cone ${ }^{-1}(n=10)$ for neurons transfected with T23N-TC10 plus L1-GFP (only one isolated fusion event was seen during the 4 min observation period in 3 of the 10 growth cones recorded). These results indicate that TC10 activity is essential for the IGF-1-triggered addition of new membrane at the growth cone of differentiating neurons (Pfenninger et al., 2003; Laurino et al., 2005).

\section{Insertion of the IGF-1 receptor}

An early event of axonal specification during neuronal differentiation is the enrichment of activatable IGF-1 receptor in one minor neurite at stage 2 of differentiation (Sosa et al., 2006). To be activated, the IGF-1 receptor needs to be inserted into the neuronal plasmalemma so that the ligand binding site is exposed to the extracellular space. Therefore, we studied the consequences of loss of function of exo70 or TC10 on the polarization of activated (i.e., phosphorylated) IGF-1 receptor [monospecificity of the antibody used for these assays has been demonstrated previously (Sosa et al., 2006)]. In stage 2 neurons transfected with a scrambled RNA sequence ( $24 \mathrm{~h}$ in vitro), deprived of growth factor for $2 \mathrm{~h}$, and stimulated for $2 \mathrm{~min}$ with $10 \mathrm{nM}$ IGF-1, we observed the expected polarized distribution of the activated IGF-1 receptor (Fig. $8 a$, top). In contrast, neurons transfected with exo70 or TC10-targeted shRNA exhibited labeling of the activated IGF-1 receptor that was less intense and not confined to any particular minor process (Fig. $8 a$, middle and bottom rows). To quantify these differences, we calculated an "active IGF-1 re- 


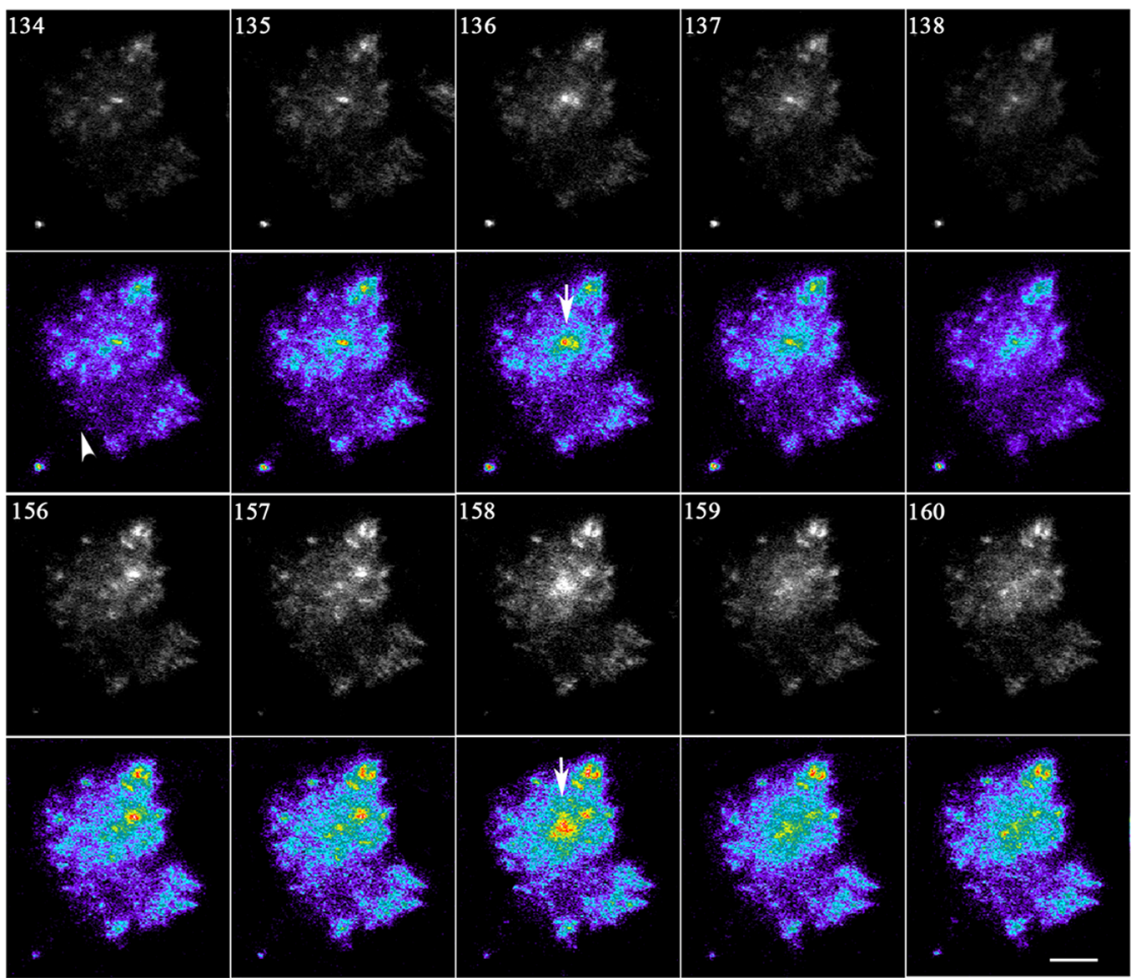

Figure 7. Time-lapse TIRF images showing two examples of fusion events in the axonal growth cone of a hippocampal pyramidal neuron transfected with wt-TC10 plus L1-GFP. Vesicles that undergo fusion are marked with arrows. Images were recorded at $1 \mathrm{~s}$ intervals, for $4 \mathrm{~min}$. Numbers indicate the time (in seconds) after the start of the series. The arrowhead indicates where the axonal shaft gives way to the growth cone. Scale bar, $1 \mu \mathrm{m}$.

ceptor polarization index" (Fig. $8 b$, legend). As shown in Figure $8 b$, this index was significantly higher $(p=0.0001)$ in the neurons transfected with the scrambled RNA sequence than in the TC10- or exo70-suppressed neurons. Neurons subjected to the same experimental conditions also were stained with $\beta g c$ antibody, which recognizes both the phosphorylated and nonphosphorylated forms of the IGF-1 receptor (Quiroga et al., 1995). Control and exo70 or TC10 expression-silenced neurons exhibited at stage 2 ( $12 \mathrm{~h}$ in culture) punctate, presumably intracellular $\beta g c$ labeling in all neurites (supplemental Fig. 6, available at www.jneurosci.org as supplemental material). After $36 \mathrm{~h}$ in culture, most control neurons had progressed to stage 3 , with all IGF-1 receptors sequestered in the axon, whereas TC10- and exo70silenced neurons failed to mature further and did not exhibit the same polarization (supplemental Fig. 7, available at www.jneurosci. org as supplemental material) (Figs. 1, 2). Together, these results suggested that TC10 and exo70 were essential for the polarized externalization of IGF-1 receptor in stage 2 neurons and, therefore, for axonal specification in hippocampal pyramidal neurons.

\section{Discussion}

During differentiation, neurons must enlarge their surface rapidly to support axonal outgrowth. This necessitates recruitment of newly synthesized membrane to the cell surface, by exocytotic insertion of PPVs at the growth cone (Pfenninger, 2009). Previous studies from our laboratories demonstrated that IGF-1, unlike the classic neurotrophins, stimulates PPV exocytosis at the axonal growth cone (Pfenninger et al., 2003). This occurs via activation of a receptor isoform that contains the immunochemically distinct $\beta$ gc subunit (Quiroga et al., 1995; Mascotti et al., 1997 ) and requires the activation of the PI3K-Akt signaling path- way (Laurino et al., 2005). Little is known, however, about (1) PPV targeting to the plasma membrane of the growth cone and (2) the relationship between membrane expansion and axonal specification for the establishment of neuronal polarity. The present report addresses these issues.

\section{Regulation of membrane expansion}

Published data suggest that the exocyst complex participates in the targeting of PPVs to neuronal plasmalemma (Hazuka et al., 1999), and mutation of the exocyst protein sec5 is known to impair addition of new membrane in developing neurons (Murthy et al., 2003). In adipocytes, the exocyst complex assembles at the plasma membrane in response to insulin, via the association of exo70 with activated TC10, and this tethers vesicles carrying Glut 4 to the site of exocytosis (Inoue et al., 2003). These observations prompted us to investigate the roles of TC10 and exo70 in IGF1-regulated membrane expansion in hippocampal neurites.

Our results show that expression of TC10 and exo70 is substantial in hippocampal neurons developing in culture. Whereas TC10 is distributed throughout the polarized neuron, exo70 is targeted selectively to the distal axon, in which the IGF-1 receptor is enriched also. Thus, the IGF-1 receptor, TC10, and exo70 colocalize at the axonal growth cone, a prominent site of membrane addition. In adipocytes and PC12 cells, insulin (Inoue et al., 2003) and NGF (Pommereit and Wouters, 2007), respectively, activate TC10, which recruits exo70 to the plasmalemma. We show here that IGF-1 (but not insulin) triggers robust and significant activation of TC10 in growth cones isolated from fetal brain. In both GCPs and hippocampal neurons in culture, TC10 activation by IGF-1 results in translocation of exo70 (and, presumably, other proteins of the exocyst complex) to the distal axonal plasma membrane. Overexpression of a dominant-negative form of TC10 abolishes this translocation, even in the presence of IGF-1. Therefore, IGF-1 stimulation and activation of TC10, in conjunction with functional exo70, are essential for membrane expansion, as shown by the recruitment of L1-GFP to the cell surface, and thus for axon elongation. Together with our previous findings (Pfenninger et al., 2003; Laurino et al., 2005), these results show that, in hippocampal pyramidal neurons in culture, membrane expansion at the axonal growth cone is regulated by IGF-1 via a cascade involving PI3K, TC10, and the exocyst complex.

\section{Membrane expansion and axon specification}

We have shown previously that IGF-1 and its receptor, which regulate exocytosis of PPVs at the axonal growth cone, are essential for the establishment of neuronal polarity (Sosa et al., 2006). IGF-1 could exert its influence on axon specification via a number of different mechanisms including pathways that do not include membrane expansion. We show here, however, that TC10- and exo70-deficient hippocampal neurons (i.e., neurons that cannot assemble the exocyst at the plasmalemma and exhibit impaired membrane expansion) are incapable of polar- 

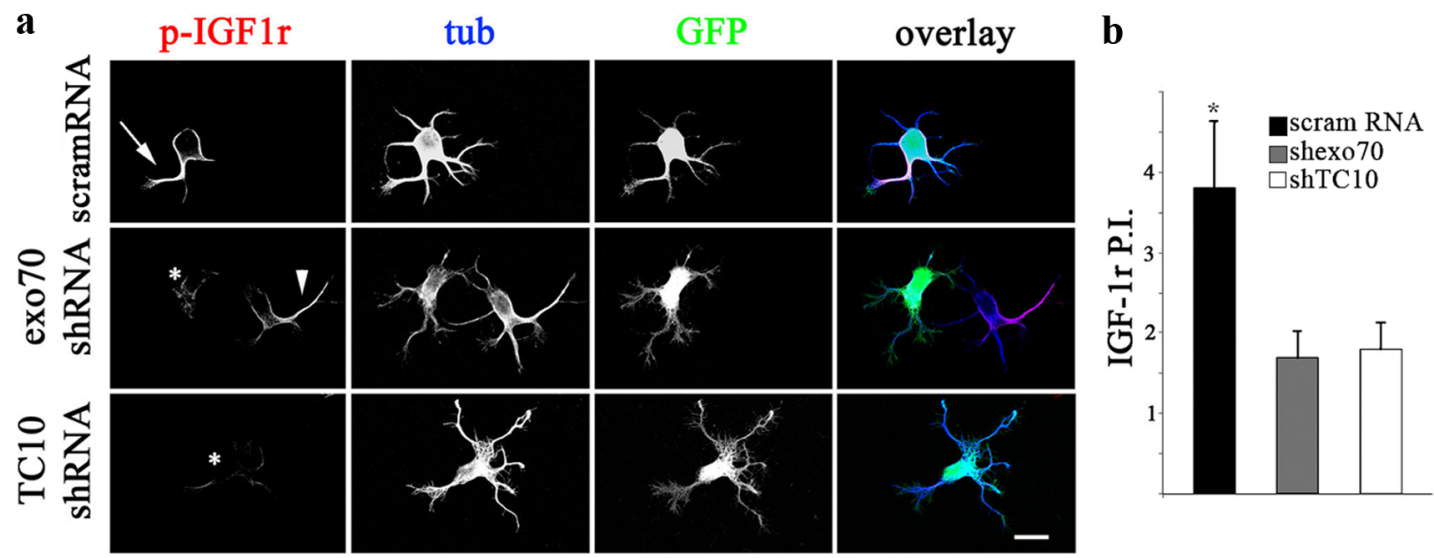

Figure 8. ex070 and TC10 loss of function inhibits the polarization of activatable IGF-1 receptor. $a$, Triple-immunofluorescence micrographs of hippocampal neurons (12 h in culture) that show the distributions of tyrosinated $\alpha$-tubulin (blue), phosphorylated IGF-1 receptor (red), and the transfection marker GFP. Neurons were transfected with either a scrambled RNA sequence (scramRNA-top), ex070-targeted shRNA (middle), or TC10 targeted shRNA (bottom). Neurons were deprived of growth factors for $4 \mathrm{~h}$ and stimulated with $10 \mathrm{~nm} \mathrm{IGF-1} \mathrm{before} \mathrm{fixation.} \mathrm{Note} \mathrm{the}$ polarization of active (membrane-inserted) IGF-1 receptor to one of the minor neurites of the cell transfected with scramRNA (arrow; top) and of a nontransfected cell (arrowhead; middle). In contrast, neurons transfected with ex070 or TC10 targeted shRNA (asterisks; middle and bottom) fail to polarize the active IGF-1 receptor. $\boldsymbol{b}$, A "polarization index" of active IGF-1 receptor (IGF-1r P.I.) was calculated as the fluorescence intensity (A.U.) of the brightest minor neurite/average fluorescence intensity (A.U.) of the other minor neurites of the same cell. Neurons were processed as in $\boldsymbol{a}$. The polarization index is significantly higher in the neurons transfected with scramRNA $\left({ }^{*} p=0.0001\right)$ compared with those transfected with ex0 70 - or TC10-targeted shRNA. Error bars indicate SEM. Scale bar, $10 \mu \mathrm{m}$.

ization. This indicates that, surprisingly, exocytosis of PPVs is necessary for axon specification.

Activation of the IGF-1 receptor requires its insertion into the plasmalemma. By probing for the appearance of activated IGF-1 receptor in undifferentiated neurites, we demonstrate that the exocyst complex is necessary for IGF-1 receptor externalization in nonpolarized neurons. It follows, therefore, that insertion of IGF- 1 receptor in an undifferentiated neurite (stage 2 hippocampal pyramidal neurons) is necessary for polarization. In other words, the TC10-exocyst complex can control axonal specification via polarized exocytosis of the IGF-1 receptor. Because IGF- 1 activates TC10 and triggers exocyst assembly, it may regulate the insertion of its own receptor (among other membrane proteins not directly related to neuronal polarization, such as L1). This is a positive-feedback mechanism that could rapidly amplify the membrane expansion response to IGF-1 and, thus, the growth rate of an undifferentiated neurite.

We propose, therefore, that the process of IGF-1 receptor mobilization to neurite plasmalemma/receptor activation and additional membrane expansion may be (one of) the selfreinforcing mechanism(s) deemed necessary for axonal specification (Arimura and Kaibuchi, 2007; Pfenninger, 2009). We do not know what mechanism confines this process to a single neurite. However, initial insertion of a few IGF-1 receptors in one of the undifferentiated neurites (possibly a stochastic process) would suffice to trigger the described positive-feedback mechanism and accelerated growth of the axon-designate.

\section{References}

Arimura N, Kaibuchi K (2007) Neuronal polarity: from extracellular signals to intracellular mechanisms. Nat Rev Neurosci 3:194-205.

Bisbal M, Conde C, Donoso M, Bollati F, Sesma J, Quiroga S, Díaz Añel A, Malhotra V, Marzolo MP, Cáceres A (2008) Protein kinase D regulates trafficking of dendritic membrane proteins in developing neurons. J Neurosci 28:9297-9308.

Chiang SH, Baumann CA, Kanzaki M, Thurmond DC, Watson RT, Neudauer CL, Macara IG, Pessin JE, Saltiel AR (2001) Insulin-stimulated GLUT4 translocation requires the CAP-dependent activation of TC10. Nature 410:944-948.

Craig AM, Banker G (1994) Neuronal polarity. Annu Rev Neurosci $17: 267-310$.
Craig AM, Wyborski RJ, Banker GA (1995) Preferential addition of newly synthetized membrane protein at axonal growth cones. Nature 375:592-594.

Dequidt C, Danglot L, Alberts P, Galli T, Choquet D, Thoumine O (2007) Fast turnover of $\mathrm{L} 1$ adhesions in neuronal growth cones involving both surface diffusion and exo/endocytosis of L1 molecules. Mol Biol Cell 8:3131-3143.

Finger FP, Hughes TE, Novick P (1998) Sec3p is a spatial landmark for polarized secretion in budding yeast. Cell 92:559-571.

Futerman AH, Banker GA (1996) The economics of neurite outgrowththe addition of new membrane to growing axons. Trends Neurosci 19: $144-149$.

Grindstaff KK, Yeaman C, Anandasabapathy N, Hsu SC, Rodriguez-Boulan E, Scheller RH, Nelson WJ (1998) Sec6/8 complex is recruited to cell-cell contacts and specifies transport vesicle delivery to the basal-lateral membrane in epithelial cells. Cell 93:731-740.

Guo W, Roth D, Walch-Solimena C, Novick P (1999) The exocyst is an effector for Sec4p, targeting secretory vesicles to sites of exocytosis. EMBO J 18:1071-1080.

Hazuka CD, Foletti DL, Hsu SC, Kee Y, Hopf FW, Scheller RH (1999) The Sec6/8 complex is located at neurites outgrowth and axonal synapseassembly domains. J Neurosci 19:1324-1334.

Inoue M, Chang L, Hwang J, Chiang SH, Saltiel AR (2003) The exocyst complex is required for targeting of Glut 4 to the plasma membrane by insulin. Nature 422:629-633.

Kawase K, Nakamura T, Takaya A, Aoki K, Namikawa K, Kiyama H, Inagaki S, Takemoto H, Saltiel AR, Matsuda M (2006) GTP hydrolysis by the Rho family GTPase TC10 promotes exocytic vesicle fusion. Dev Cell 3:411421.

Laurino L, Wang XX, de la Houssaye BA, Sosa L, Dupraz S, Cáceres A, Pfenninger KH, Quiroga S (2005) PI3K activation by IGF-1 is essential for the regulation of membrane expansion at the nerve growth cone. J Cell Sci 118:3653-3662.

Lohse K, Helmke SM, Wood MR, Quiroga S, de la Houssaye BA, Miller VE, Negre-Aminou P, Pfenninger KH (1996) Axonal origin and purity of growth cones isolated from fetal rat brain. Dev Brain Res 96:83-96.

Mascotti F, Cáceres A, Pfenninger KH, Quiroga S (1997) Expression and distribution of IGF-1 receptors containing a $\beta$-subunit variant $(\beta \mathrm{gc})$ in developing neurons. J Neurosci 4:1447-1459.

Ménager C, Arimura N, Fukata Y, Kaibuchi K (2004) PIP3 is involved in neuronal polarization and axon formation. J Neurochem 89:109-118.

Murthy M, Garza D, Scheller RH, Schwarz TL (2003) Mutations in the exocyst component Sec5 disrupt neuronal membrane traffic, but neurotransmitter release persists. Neuron 37:433-447.

Nishimura T, Yamaguchi T, Kato K, Yoshizawa M, Nabeshima Y, Ohno S, 
Hoshino M, Kaibuchi K (2005) PAR-6-PAR-3 mediates Cdc42-induced Rac activation through the Rac GEFs STEF/Tiam. Nat Cell Biol 7:270-277.

Paglini G, Kunda P, Quiroga S, Kosik K, Cáceres A (1998) Suppression of radixin and moesin alters growth cone morphology, motility, and process formation in primary cultured neurons. J Cell Biol 143:443-455.

Pfenninger KH (2009) Plasma membrane expansion: a neuron's Herculean task. Nat Rev Neurosci 4:251-261.

Pfenninger KH, Friedman LB (1993) Sites of plasmalemmal expansion in growth cones. Dev Brain Res 71:181-192.

Pfenninger KH, Maylié-Pfenninger MF (1981) Lectin labelling of sprouting neurons. II. Relative movement and appearance of glyconjugates during plasmalemmal expansion. J Cell Biol 89:547-559.

Pfenninger KH, Ellis L, Johnson MP, Friedman LB, Somlo S (1983) Nerve growth cones isolated from fetal rat brain: subcellular fractionation and characterization. Cell 35:573-584.

Pfenninger KH, Laurino L, Peretti D, Wang X, Rosso S, Morfini G, Cáceres A, Quiroga S (2003) Regulation of membrane expansion at the nerve growth cone. J Cell Sci 116:1209-1217.

Pommereit D, Wouters FS (2007) An NGF-induced Exo70-TC10 complex locally antagonises Cdc42-mediated activation of N-WASP to modulate neurite outgrowth. J Cell Sci 120:2694-2705.

Quiroga S, Garofalo RS, Pfenninger KH (1995) Insulin-like growth factor I receptors of fetal brain are enriched in nerve growth cones and contain a $\beta$-subunit variant. Proc Natl Acad Sci U S A 92:4309-4312.

Rosso S, Bollati F, Bisbal M, Peretti D, Sumi T, Nakamura T, Quiroga S, Ferreira A, Cáceres A (2004) LIMK1 regulates Golgi dynamics, traffic of
Golgi-derived vesicles, and process extension in primary cultured neurons. Mol Biol Cell 15:3433-3449.

Sans N, Prybylowski K, Petralia RS, Chang K, Wang YX, Racca C, Vicini S, Wenthold RJ (2003) NMDA receptor trafficking through an interaction between PDZ proteins and the exocyst complex. Nat Cell Biol 5:520-530.

Shi SH, Jan LY, Jan YN (2003) Hippocampal neuronal polarity specified by spatially localized mPar3/mPar6 and PI 3-kinase activity. Cell 112:63-75.

Sosa L, Dupraz S, Laurino L, Bollati F, Bisbal M, Cáceres A, Pfenninger KH, Quiroga S (2006) IGF-1 receptor is essential for the establishment of hippocampal neuronal polarity. Nat Neurosci 8:993-995.

TerBush DR, Novick P (1995) Sec6, Sec8, and Sec15 are components of a multisubunit complex which localizes to small bud tips in Saccharomyces cerevisiae. J Cell Biol 130:299-312.

TerBush DR, Maurice T, Roth D, Novick P (1996) The exocyst is a multiprotein complex required for exocytosis in Saccharomyces cerevisiae. EMBO J 15:6483-6494.

Vega IE, Hsu SC (2001) The exocyst complex associates with microtubules to mediate vesicle targeting and neurite outgrowth. J Neurosci 21:38393848

Xia XG, Zhou H, Ding H, Affar el B, Shi Y, Xu Z (2003) An enhanced U6 promoter for synthesis of short hairpin RNA. Nucleic Acids Res 31:e100

Yeaman C, Grindstaff KK, Wright JR, Nelson WJ (2001) Sec6/8 complex on trans-Golgi network and plasma membrane regulate late stages of exocytosis in mammalian cells. J Cell Biol 155:593-604.

Yudowski GA, Puthenveedu MA, von Zastrow M (2006) Distinct modes of regulated receptor insertion to the somatodendritic plasma membrane. Nat Neurosci 9:622-627. 